\title{
Habilidades sociais, problemas de comportamento e competência acadêmica de crianças de famílias nucleares e recasadas $^{1}$
}

\section{Social skills, behavior problems and academic competence by children of nuclear and remarried families}

\section{Habilidades sociales, problemas de comportamiento y rendimiento escolar de los niños de las famílias tradicionales y reconstituidas}

\author{
Vanessa Barbosa Romera Leme* \\ Universidade Salgado de Oliveira - Universo, Niterói, Rio de Janeiro, Brasil
}

\section{Edna Maria Marturano**}

Universidade de São Paulo - Faculdade de Medicina - FMRP, Ribeirão Preto, São Paulo, Brasil

\section{Anne Marie Germaine Victorine Fontaine*** \\ Universidade do Porto - FPCEUP, Porto, Portugal}

\begin{abstract}
RESUMO
A partir das percepções de mães e de professoras, este estudo tem por objetivo avaliar semelhanças e diferenças nas habilidades sociais, problemas de comportamento e competência acadêmica de crianças de famílias nucleares e recasadas. Participaram 94 mães (33 de famílias nucleares, 31 recasadas há menos de três anos e 30 recasadas há mais de três anos), cujos filhos passavam pela transição para o primeiro ano do Ensino Fundamental, e 22 professoras das crianças. 0 instrumento utilizado foi 0 Sistema de Avaliação de Habilidades Sociais (versão pais e professores). 0 tipo de família não foi associado às habilidades sociais nem à competência acadêmica das crianças, mas apenas aos comportamentos externalizantes. De forma geral, segundo os relatos das mães, as crianças de famílias nucleares apresentaram mais habilidades sociais e menos problemas de comportamento que as crianças de famílias recasadas. Para as professoras, não houve diferenças nas habilidades sociais e na competência acadêmica das crianças de famílias nucleares e recasadas. Contudo, segundo as avaliações das professoras, as crianças de famílias recasadas apresentam mais comportamentos externalizantes quando comparadas às crianças de famílias nucleares.

Palavras-chave: pais, crianças, família, comportamento, competência.
\end{abstract}

\section{ABSTRACT}

From the perceptions about mothers and teachers, this study aims to assess similarities and differences in social skills, behavior problems and academic 
competence of children from nuclear families and stepfamilies. Participants are 94 mothers (33 nuclear family mothers, 31 mothers who have been remarried for less than three years and 30 mothers who have been remarried for over three years), whose children passed through the transition of first grade of elementary school, and 22 teachers. The instrument used is Social Skills Rating System (version parents and teachers). In general, according to the reports of mothers, children of nuclear families had more social skills and fewer behavior problems than children of stepfamilies. For teachers, there were no differences in social skills and academic competence of children from nuclear and stepfamilies. However, according to evaluations of teachers, children from stepfamilies exhibit more behavior problems compared to children from nuclear families.

Keywords: parent, children, family, behavior, competence.

\begin{abstract}
RESUMEN
Desde la percepción de las madres y los maestros, este estudio tiene como objetivo evaluar similitudes y diferencias en las habilidades sociales, problemas de comportamiento) y rendimiento escolar de los niños en las familias tradicionales y familias reconstituidas. Participaran 94 madres (33 de las familias tradicionales, 31 de las familias reconstituidas con menos de tres años y 30 de las familias reconstituidas con más de tres años), cuyos hijos pasaran por la transición al primer año de la escuela primaria, y 22 maestros de los niños. El instrumento utilizado fue el Sistema de Evaluación de Las Habilidades Sociales (versión padres y maestros). En general, de acuerdo con los informes de las madres, los niños en las familias tradicionales tenían más habilidades sociales y menos problemas de comportamiento que los niños de las familias reconstituidas. Para los maestros, no hubo diferencias en las habilidades sociales y rendimiento escolar de los niños de familias nucleares y reconstituidas. Sin embargo, de acuerdo a las evaluaciones de los maestros, los niños de familias reconstituidas presentan más conductas de externalización en comparación con los niños de las familias nucleares.
\end{abstract}

Palabras clave: padres, niños, familia, conducta, rendimiento.

\title{
1 I ntrodução
}

A família brasileira encontra-se num momento de profundas mudanças em seus arranjos. O número de separações conjugais e de recasamentos aumentou consideravelmente na última década. Segundo o Instituto Brasileiro de Geografia e Estatística (IBGE, 2010), em relação ao Censo Demográfico 2000, a proporção de pessoas divorciadas praticamente dobrou, passando de $1,7 \%$, em 2000, para 3,1\% em 2010. Como consequência, alterações nos tipos de uniões, nas suas durações e nos seus términos atingiram o modelo de família nuclear formada por pai, mãe e filhos da primeira união conjugal (Cano, Gabarra, Moré, \& Crepaldi, 2009). Embora seja muito provável que tanto homens quanto mulheres voltem a se casar e ter filhos, o recasamento ainda é menos investigado quando comparado ao divórcio tanto no contexto brasileiro (Oliveira, Siqueira, 
Dell 'Agllio, \& Lopes, 2008) quanto em outros países (Anderson \& Greene, 2013; Sweeney, 2010).

No que diz respeito às uniões conjugais heterossexuais que se formam depois da separação conjugal, verifica-se que há tanto uma diversidade de configurações quanto de denominações encontradas na literatura e na sociedade para esse tipo de família (Lima, 2008; McGoldrick \& Carter, 2008/1989; Silva, Trindade, \& Silva Junior, 2012). Segundo McGoldrick e Carter (2008/1989), essas famílias são referidas de diversas formas, tais como reconstituídas, reestruturadas, "misturadas" e recasadas. No presente estudo, adotaremos o termo "recasada" para as famílias formadas por mães que tenham pelo menos um filho biológico da união anterior, e por um parceiro (padrasto), que pode ou não trazer filhos de outros relacionamentos. As famílias formadas por mães e por pais com filhos biológicos da primeira união conjugal serão referidas como famílias nucleares. Para todos os tipos de famílias serão consideradas tanto as uniões civis quanto as consensuais, ou seja, aquelas em que os cônjuges coabitam formando um casal sem formalização legal.

\section{Indicadores do ajustamento infantil frente ao recasamento}

Conforme a literatura, o recasamento parental, muitas vezes, pode gerar diversas alterações no convívio familiar decorrentes da mudança de residência para a família e de escola para os filhos, e da adaptação ao novo membro familiar (padrasto ou madrasta), às novas rotinas e regras da família e ao convívio com meio-irmãos ou filhos do(a) parceiro(a) da mãe e do pai (Cano, Gabarra, Moré, \& Crepaldi, 2009; Silva, Trindade, \& Junior, 2012; Sweeney, 2010; Wallerstein, Lewis, \& Rosenthal, 2013). Pesquisas indicam que o novo casal enfrenta diversos desafios por ter de lidar ao mesmo tempo com as demandas da nova união conjugal e com os cuidados dos filhos biológicos e enteados (Lima, 2008; King \& DeLongis, 2013).

Para alguns autores a existência de conflito entre os filhos e os adultos (tais como, pai/mãe biológico e padrasto/madrasta) pode levar a problemas de ajustamento infantil (Jensen \& Shafer, 2013; Jeynes, 2006). Os filhos enfrentariam várias dificuldades, tais como aceitar o novo relacionamento da mãe/do pai devido a sentimentos de lealdade com o outro progenitor e rivalidade com meio-irmãos ou filhos do parceiro da mãe/do pai que venham, porventura, a residir com a criança (Costa \& Dias, 2012; Ramires, 2004). Essas situações prejudicariam a construção do relacionamento entre a criança e o padrasto/madrasta e fariam com que os filhos de famílias recasadas apresentassem resultados desenvolvimentais inferiores quando comparadas às crianças de famílias nucleares (Jensen \& Shafer, 2013; Jeynes, 2006). Assim, a ocorrência desses eventos 
estressantes pode impactar negativamente os padrões de autoridade, comunicação e práticas de socialização parental (Heterington, 2003; McGoldrick \& Carter, 2008/1995). Desse modo, dependendo da idade e do gênero da criança, esta poderia, por exemplo, ter dificuldade em se adaptar às práticas disciplinares do padrasto/madrasta, além de enfrentar conflito de lealdade para com o pai/mãe biológico, aumentando a probabilidade de os filhos apresentarem problemas comportamentais, emocionais e escolares (Hetherington, 2003; Jeynes, 2006; McGoldrick \& Carter, 2008/1989, Ramires, 2004).

Apesar de resultados divergentes, diversas pesquisas encontraram que, comparadas às crianças de famílias nucleares, as que passaram pelo recasamento dos pais apresentam mais dificuldades nos relacionamentos sociais, mais problemas de comportamento internalizantes e externalizantes e menor desempenho acadêmico (Dessen \& Szelbracikowski, 2004; Hetherington, 2003; Jenkins, Simpson, Dunn, Rasbash, \& O'Connor, 2005, Ram \& Hou, 2003; Steele, Sigle-Rushton, \& Kravdal, 2009). Contudo, as relações entre o recasamento dos pais e dificuldades comportamentais e/ou menor desempenho acadêmico dos filhos devem ser analisadas como um processo dinâmico e não linear (Hetherington, 2003; Sweeney, 2010; Wallerstein, Lewis, \& Rosenthal, 2013). Os efeitos das transições familiares sobre os filhos tendem a ser indiretos e mediados por variáveis como tempo do recasamento, práticas parentais, conflito conjugal e características individuais dos pais e dos filhos, tais como idade, gênero e habilidades sociais (Anderson \& Greene, 2013; Silva et al., 2012; Sweeney, 2010). Em um estudo de revisão, Anderson e Greene (2013) chamam a atenção para o fato de que as diferenças médias verificadas entre as crianças de famílias cujos pais permaneceram casados e as crianças cujos pais formaram uma nova união conjugal depois do desenlace matrimonial são, na maior parte das vezes, modestas, havendo poucas diferenças significativas entre elas.

Outra questão relacionada às transições familiares refere-se à duração dos seus efeitos sobre dimensões do desenvolvimento infantil. Alguns autores afirmam que imediatamente após a separação ou o recasamento verifica-se um desequilíbrio nas relações familiares, seguido de uma gradual estabilização das relações para a nova formação familiar (Anderson \& Greene, 2013; Costa \& Dias, 2012; Sweeney, 2010). Esses autores consideram as transições familiares como um evento que leva a uma crise temporária nas relações familiares. Estudos encontraram que a estabilidade familiar é alcançada depois de um período de cerca de dois a três anos após o recasamento (Booth \& Amato, 2001; Hetherington, Bridges, \& Insabella, 1998), enquanto outros afirmam que essa estabilidade pode demorar entre três e cinco anos para ocorrer (Lima, 2008). Conforme McGoldrick e Carter (2008/1995), esse tempo não é muito 
preciso porque dependerá, por exemplo, do grau de conflito entre os ex-cônjuges, do tempo entre a separação e o recasamento e da idade dos filhos.

Todavia, algumas pesquisas têm sinalizado certa persistência dos efeitos negativos causados pelas transições familiares em outras fases do ciclo vital (Jenkins, Simpson, Dunn, Rasbash, \& O'Connor, 2005) ou mesmo gerações (Amato, 2005). Algumas pesquisas longitudinais têm identificado a existência de discórdias nas interações entre os membros da nova formação familiar que não são resolvidas nos primeiros anos do recasamento e que podem ressurgir ou mesmo gerar novas tensões anos mais tarde (Hetherington, Bridges, \& Insabella, 1998; King \& DeLongis, 2013; Wallersteins et al., 2013). Nesse sentido, Hetherington (1993) observou que as mães recentemente recasadas relataram mais satisfação conjugal que as mães que se mantiveram casadas, o que a autora denominou de período de lua de mel (honeymoon). Assim, o clima conjugal mais harmonioso poderia contribuir para relações mais afetuosas entre mães/padrastos e filhos, mas com o tempo dificuldades de definições quanto aos papéis parentais e conflitos de lealdade podem surgir e prejudicar as interações entre mães e filhos, repercutindo no comportamento social e acadêmico dos últimos. Portanto, os resultados de pesquisa acerca das dificuldades apresentadas pelas crianças em resposta ao recasamento dos pais são pouco conclusivos (Hetherington, 2003; Oliveira, Siqueira, Dell'Aglio, \& Lopes, 2008; Sweeney, 2010), sugerindo uma área de conhecimento que merece ser mais investigada. Além disso, embora algumas pesquisas (Amato, 2005; Hetherington, 2003; Jeynes, 2006) indiquem que 0 recasamento pode interferir nas relações das crianças em outros ambientes, por exemplo, as relações da criança com amigos e professores da escola, é importante que os estudos procurem analisar e diferenciar essa possível influência sobre diversos aspectos do desenvolvimento infantil. O contexto escolar pode oferecer fatores de proteção que ajudam a criança na gestão das dificuldades vividas no ambiente da família (Marturano, Elias, \& Leme, 2012).

\section{Recasamento, transição para o primeiro ano do ensino fundamental e habilidades sociais}

O ingresso da criança no primeiro ano do Ensino Fundamental é uma mudança normativa significativa que se configura como um período de imprevisibilidades e incertezas que podem gerar estresse e ansiedade para a criança (Marturano, 2008; Stasiak \& Weber, 2013). Isso ocorreria porque quando a criança entra no Ensino Fundamental há a necessidade de aprendizagem de novas habilidades acadêmicas e sociais, pois a criança é exposta a um conjunto de situações (tais 
como as novas relações com os pares e com os adultos do contexto escolar) que transcendem o ambiente familiar (Trivellato-Ferreira \& Marturano, 2008). Há, então, a necessidade de lidar com as expectativas do meio social que se tornam mais exigentes, em um ambiente onde a dependência é menos tolerada e há menos suporte (Marturano, 2008). E este momento de transição, pelo qual a criança passa, vai demandar novas habilidades dos pais em supervisionar e monitorar as recentes experiências dos filhos na escola, bem como perceber qualquer dificuldade com as novas aprendizagens e contatos sociais (Stasiak \& Weber, 2013).

Pesquisas têm indicado que a transição para o primeiro ano pode ser facilitada quando há estabilidade no ambiente familiar, suporte parental e as relações entre pais e filhos caracterizadas por interações com afeto positivo, reciprocidade e equilíbrio de poder (Cadima, McWilliam, \& Leal, 2010; Hoglund \& Leadbeart, 2004; Trivellato-Ferreira \& Marturano, 2008). Por sua vez, o recasamento parental parece prejudicar justamente esses processos por ser um momento de vida em que há maior probabilidade de ocorrência de instabilidade no ambiente das famílias e utilização de práticas educativas inadequadas (Amato, 2005; Jeynes, 2006; Hetherington et al., 1998). Portanto, a sobreposição de transições, como o fato de a criança estar vivenciando a passagem para o Ensino Fundamental e tendo de lidar, ao mesmo tempo, com as alterações no contexto familiar decorrentes do recasamento, poderia ser demasiado exigente para a criança. Tal sobrecarga, por seu turno, poderia prejudicar a realização das tarefas do desenvolvimento requeridas na idade escolar (Jeynes, 2006; Hoglund \& Leadbeart, 2004). Nesse sentido, habilidades sociais, entendidas como um conjunto de comportamentos (por exemplo, expressar sentimentos, defender direitos, oferecer ajuda, acalmar-se diante de situações-problema etc.) que a criança emite num determinado contexto sociocultural (Caballo, 2010; Del Prette \& Del Prette, 2005), podem ajudá-la a se relacionar de maneira positiva com pares e professores e auxiliá-la na transição ao primeiro ano do Ensino Fundamental.

A separação e o recasamento parental não são em si eventos de vida associados às trajetórias desenvolvimentais menos favoráveis na infância (Anderson \& Greene, 2013; Silva et al., 2012). Em vez disso, é a interconexão complexa entre os recursos pessoais da criança e do contexto disponíveis que influencia a forma como pais e filhos lidam com os desafios das transições familiares, mas também aproveitam as oportunidades que surgem durante as continuidades $e$ descontinuidades ao longo do ciclo de vida. Ao assumir essa perspectiva, rompe-se com o modelo de déficit encontrado em muitos estudos sobre recasamento, que focam apenas os problemas socioemocionais e comportamentais dos filhos (Hetherington et al., 1998). Dessa forma, neste estudo, procura-se destacar que as 
crianças de famílias recasadas podem, inclusive, ter habilidades sociais diferenciadas, uma vez que as demandas requeridas e as relações interpessoais estabelecidas no ambiente da família recasada produzem processos relacionais únicos. Assim, competências interpessoais, tais com as habilidades sociais em crianças de famílias recasadas, podem funcionar como fatores de proteção. Tais recursos, uma vez identificados por meio de pesquisa empírica, podem ser desenvolvidos em intervenções psicológicas com aquelas que apresentam dificuldades.

A partir dessas considerações, segundo as percepções de mães e de professoras, o presente estudo teve por objetivo avaliar semelhanças e diferenças em alguns indicadores de adaptação, tais como as habilidades sociais, os problemas de comportamento e a competência acadêmica de crianças de famílias nucleares e recasadas, durante a transição para o primeiro ano do Ensino Fundamental. Tem-se por hipóteses que as crianças de famílias nucleares e recasadas estáveis apresentam mais habilidades sociais e competência acadêmica e menos problemas de comportamento em comparação com as crianças de famílias recasadas recentes. As hipóteses do estudo apoiam-se em pesquisas que sinalizam que os efeitos das transições familiares são temporários, ou seja, com o passar do tempo (em torno de três anos após o recasamento), as famílias ajustam-se às novas configurações e as crianças deixam de apresentar as dificuldades emocionais e/ou comportamentais evidenciadas logo após o recasamento (Booth \& Amato, 2001; Heterington et al., 1998).

\section{Método}

\section{Participantes}

O estudo seguiu um delineamento correlacional com comparação de grupos. A amostra selecionada por conveniência foi composta por 94 mães (33 mães de famílias nucleares, 31 mães de famílias recasadas em transição recente e 30 mães de famílias recasadas estáveis), cujos filhos frequentavam o primeiro ano do Ensino Fundamental. Os alunos estavam matriculados em cinco escolas da rede municipal de uma cidade do interior do Estado de São Paulo. O número de participantes em cada grupo atendeu a um dos requisitos para análise estatística paramétrica, no pressuposto de que em amostras com 25-30 participantes a distribuição da média amostral é próxima à normal (Marôco, 2011). A verificação do outro requisito, o de normalidade das distribuições das variáveis nos grupos, é descrita adiante, na seção Procedimento de análise de dados. As mães (idade média de 32 anos, DP $=5,9$ ) tinham em média, 8,6 anos de 
escolaridade ( $D P=2,8)$. As crianças (48 meninas e 46 meninos) tinha idade entre seis e sete anos. As famílias do estudo encontravam-se entre a classe $\mathrm{C} 1$ e a classe $\mathrm{C} 2$, o que corresponde a $48 \%$ da distribuição da população do Estado de São Paulo (Associação Brasileira e Empresas de Pesquisa [ABEP], 2010). Quando as mães recasaram as crianças das famílias recasadas recentes tinham em média 5,3 anos (DP $=0,83$ ) e as crianças de famílias recasadas estáveis tinham em média $2,7(D P=0,78)$. Todas as mães de famílias recasadas tinham a guarda exclusiva dos filhos.

Para participar do estudo, como critério de inclusão, as mães deveriam residir com as crianças porque elas iriam avaliar os comportamentos dos filhos. Outros critérios de inclusão referendados na literatura (Hetherington, 1993; Jeynes, 2006) foram estabelecidos para definir as famílias nucleares e recasadas em transição recente e estável, assim como a recência do recasamento: (1) Famílias nucleares: quando a mãe biológica da criança coabitava numa união civil ou por consenso com o pai biológico da criança desde o nascimento desta; (2) Famílias recasadas em transição recente e estável: quando a mãe biológica da criança coabitava numa união civil ou por consenso com outro parceiro, que não era o pai biológico da criança; a mãe deveria ter coabitado com o ex-cônjuge (pai biológico da criança) durante no mínimo um ano, a contar do nascimento da criança. Para a família ser considerada como recasada em transição recente, a mãe deveria estar recasada há pelo menos um ano e há menos de três anos; para ser considerada como família recasada estável, a mãe deveria estar recasada há mais de três anos. Participaram, ainda, do estudo 22 professoras (idade média 41 anos, $\mathrm{DP}=10,48)$ do primeiro ano em cinco escolas. As professoras lecionavam apenas no ensino regular, em média há 12 anos (DP = $2,16)$. Todas haviam concluído o ensino superior.

Instrumentos

Sistema de Avaliação de Habilidades Sociais (Social Skills Rating System, SSRS-BR) - versão para pais e professores. É um instrumento que avalia o repertório de habilidades sociais, problemas de comportamento e competência acadêmica das crianças (esta última apenas na versão para professores) que foi validado para o Brasil (SSRS-BR, Bandeira, Del Prette, Del Prette, \& Magalhães, 2009). As versões para pais e professores são bastante semelhantes. A frequência com que a criança apresenta habilidades sociais (por exemplo, cooperação, autocontrole e assertividade), problemas de comportamento (hiperatividade, externalizante e internalizante) e competência acadêmica é avaliada em escalas de três pontos (nunca, às vezes, sempre). Na amostra de validação, foram encontrados índices aceitáveis de consistência interna e estabilidade temporal. No 
presente estudo, em que foram utilizadas as versões de pais (alfa entre 0,83 e 0,86 na amostra de validação) e professores (alfa entre 0,91 e 0,94 na amostra de validação), foram encontrados valores de alfa acima de 0,73 nas duas versões, exceto para o componente Internalizantes da escala de comportamentos problemáticos, versão para pais ( $a=0,37)$; assim, esta dimensão não foi incluída nas análises.

Questionário de Caracterização Familiar. Foi um instrumento elaborado para este estudo para obter informações tais como idade e sexo das crianças e das mães, moradia e o estado civíl das mães (por exemplo, tempo de casamento, separação e/ou recasamento).

\section{Procedimento}

Coleta de dados

O projeto foi aprovado por Comitê de Ética em Pesquisa, de acordo com o Processo $n^{\circ}$ 379/2008-2008.1.449.59.7. Para a seleção da amostra, após assinarem o Termo de Consentimento Livre e Esclarecido (TCLE), as professoras foram solicitadas a mandar, no caderno de recado dos alunos, um comunicado às mães, solicitando um meio de contato e perguntando o seu estado civil (casada, separada ou recasada). Em posse das respostas, a pesquisadora entrava em contato com as mães, por telefone, para verificar o interesse em participar do estudo e conferir os critérios de inclusão. Em seguida, havendo interesse das mães, a pesquisadora marcava um encontro para a assinatura do TCLE e para a aplicação dos instrumentos. A coleta de dados com as mães foi realizada nas suas residências ou locais de trabalho. Mediante a assinatura do TCLE, a pesquisadora aplicava os instrumentos: Questionário de Caracterização Familiar, SSRS-BR e Questionário Classificação Econômica Brasil. Após a coleta com as mães, a pesquisadora agendou com cada professora um horário para que avaliasse as crianças. A pesquisadora aplicou com cada professora, individualmente, o SSRS-BR, na própria escola, após o término das aulas.

Análise de dados

A análise de dados foi realizada no programa SPSS 18.0 e compreendeu duas etapas. Primeiramente foi verificado 0 pressuposto de normalidade para a realização de análises paramétricas, por meio do teste Kolmogorov-Smirnov. Em seguida, para as variáveis com distribuição normal, os grupos foram comparados por meio de análises de variância (ANOVA one-way), 
com o teste post hoc de Bonferroni, em caso de homogeneidade das variáveis, ou o teste Dunnett's T3, em caso de heterogeneidade. Para variáveis com distribuição não normal, as comparações foram feitas com o teste de Kruskal-Wallis, utilizando-se o teste Post Hoc LSD de Fisher, de acordo com a recomendação de Marôco (2011). Segundo o autor, o teste Kruskal-Wallis não permite saber em quais grupos de famílias as distribuições das variáveis avaliadas diferem significativamente. Assim, uma alternativa é converter as observações em ordens usando a função Transform (Rank Cases) do SPSS e, em seguida, realizar uma ANOVA one-way sobre a nova variável das ordens com a comparação múltipla de médias das ordens pelo método LSD de Fisher.

\section{Resultados}

A Tabela 1 apresenta os resultados a respeito das habilidades sociais e dos problemas de comportamento das crianças, do ponto de vista das mães de famílias nucleares e recasadas. 
Tabela 1

Habilidades Sociais e Problemas de Comportamento das crianças: resultados das comparações entre as famílias nucleares e recasadas, segundo a avaliação das mães

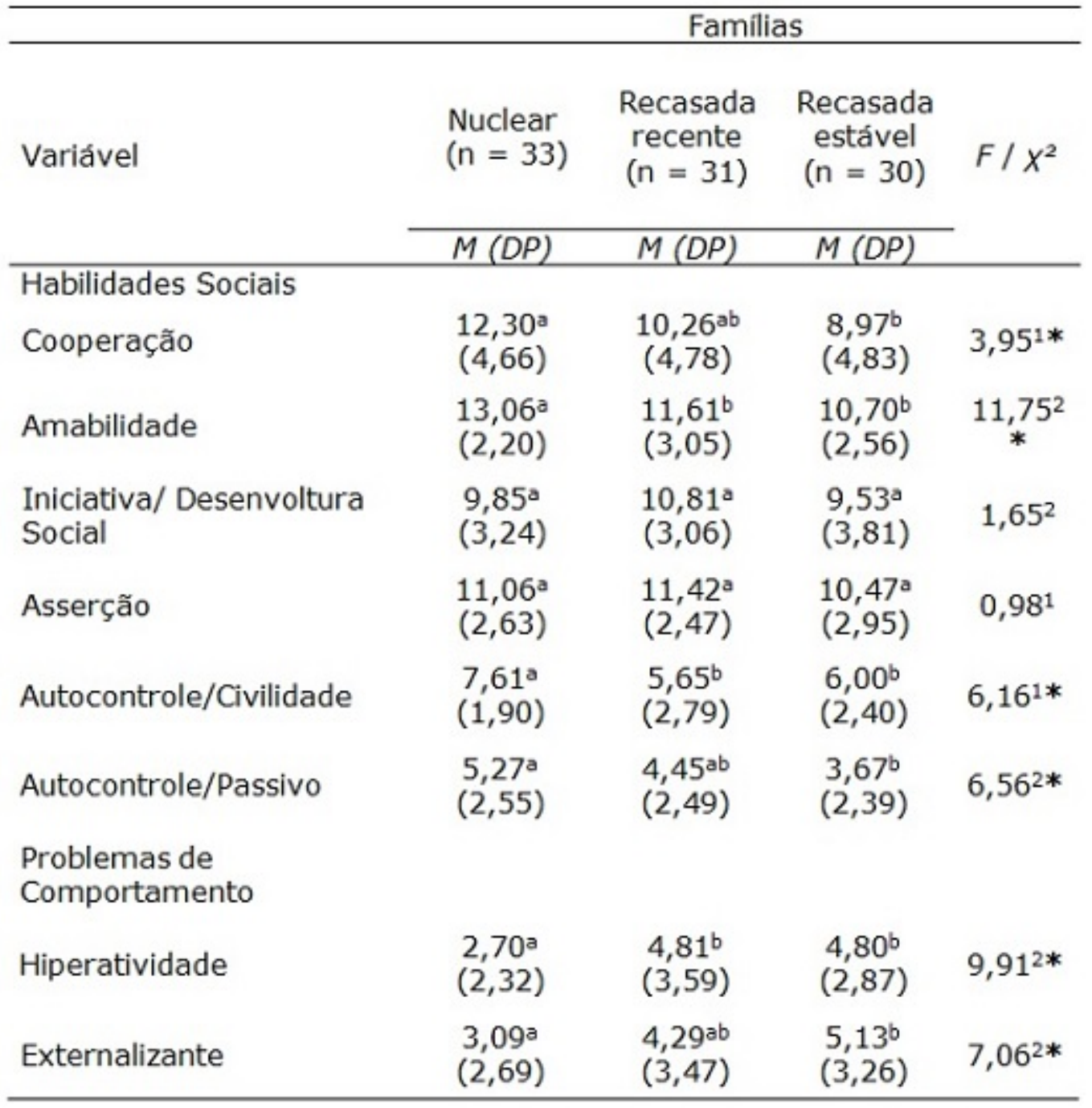

Nota: Diferentes letras simbolizam diferenças entre as familias ( $\mathrm{N}=$ 94); ${ }^{1}$ ANOVA: variável com distribuição normal; ${ }_{w}^{2}$ Teste $\mathrm{H}$ de KruskalWallis: variável com distribuição não normal.

$* p \leq 0,05$

Constata-se pela Tabela 1 , que as mães de famílias nucleares relataram que seus filhos apresentavam com mais frequência, em comparação com as crianças de famílias recasadas recentes e estáveis, habilidades sociais de Amabilidade e de Autocontrole/Civilidade. Além disso, as crianças de famílias nucleares foram percebidas pelas mães como apresentando mais habilidades de Cooperação e de Autocontrole/Passivo, em comparação com as crianças de famílias recasadas estáveis, mas não recentes. Para todas as habilidades sociais investigadas, as crianças de famílias recasadas 
recentes e recasadas estáveis não se diferenciaram. As crianças dos diferentes tipos de famílias não apresentam diferenças nas habilidades sociais de Iniciativa/Desenvoltura Social e de Asserção. No que diz respeito aos problemas de comportamento, as mães de famílias recasadas recentes e estáveis perceberam que seus filhos apresentavam mais problemas comportamentais do tipo hiperativo que as crianças de famílias nucleares. Além disso, as crianças de famílias recasadas estáveis apresentaram mais problemas de comportamento externalizantes que as crianças de famílias nucleares. As crianças de famílias recasadas recentes e estáveis não se diferenciaram para os problemas de comportamento investigados.

A Tabela 2 apresenta os resultados a respeito das habilidades sociais, dos problemas de comportamento e da competência acadêmica das crianças de famílias nucleares e recasadas, segundo a avaliação das professoras. 
Tabela 2

Habilidades Sociais e Problemas de Comportamento das crianças: resultados das comparações entre as famílias nucleares e recasadas, segundo a avaliação das professoras

\begin{tabular}{|c|c|c|c|c|}
\hline \multirow{3}{*}{ Variável } & \multicolumn{4}{|c|}{ Familias } \\
\hline & $\begin{array}{l}\text { Nuclear } \\
(n=33)\end{array}$ & $\begin{array}{l}\text { Recasada } \\
\text { recente } \\
(n=31)\end{array}$ & $\begin{array}{c}\text { Recasada } \\
\text { estável } \\
(n=30)\end{array}$ & $F / X^{2}$ \\
\hline & $M(D P)$ & $M(D P)$ & $M(D P)$ & \\
\hline \multicolumn{5}{|l|}{ Habilidades Sociais } \\
\hline $\begin{array}{l}\text { Responsabilidade/Coope } \\
\text { ração }\end{array}$ & $\begin{array}{l}15,94^{\circ} \\
(4,38)\end{array}$ & $\begin{array}{l}14,19^{a} \\
(5,18)\end{array}$ & $\begin{array}{l}14,57^{a} \\
(4,43)\end{array}$ & $3,44^{2}$ \\
\hline Asserção Positiva & $\begin{array}{l}12,94^{a} \\
(4,10)\end{array}$ & $\begin{array}{l}12,68^{a} \\
(4,44)\end{array}$ & $\begin{array}{l}12,43^{a} \\
(3,86)\end{array}$ & $0,46^{2}$ \\
\hline Autocontrole & $\begin{array}{l}11,58^{a} \\
(3,59)\end{array}$ & $\begin{array}{l}9,32^{a} \\
(3,77)\end{array}$ & $\begin{array}{l}10,07^{a} \\
(3,87)\end{array}$ & $3,05^{1}$ \\
\hline Autodefesa & $\begin{array}{l}3,91^{a} \\
(1,60)\end{array}$ & $\begin{array}{l}4,00^{\circ} \\
(1,77)\end{array}$ & $\begin{array}{r}4,07^{\circ} \\
(1,57)\end{array}$ & $0,30^{2}$ \\
\hline Cooperação com Pares & $\begin{array}{l}6,03^{a} \\
(1,94)\end{array}$ & $\begin{array}{l}5,48^{a} \\
(2,14)\end{array}$ & $\begin{array}{l}5,47^{\mathrm{a}} \\
(1,94)\end{array}$ & $1,70^{2}$ \\
\hline \multicolumn{5}{|l|}{$\begin{array}{l}\text { Problemas de } \\
\text { Comportamento }\end{array}$} \\
\hline Externalizante & $\begin{array}{l}4,12^{\mathrm{a}} \\
(5,02)\end{array}$ & $\begin{array}{l}8,23^{b} \\
(6,42)\end{array}$ & $\begin{array}{l}7,80^{\mathrm{b}} \\
(5.33)\end{array}$ & $\begin{array}{c}11,80^{2} \\
*\end{array}$ \\
\hline Competência Acadêmica & $\begin{array}{l}33,15 \\
(10,17)\end{array}$ & $\begin{array}{c}31,03 \\
(11,06)\end{array}$ & $\begin{array}{l}32,07 \\
(8,65)\end{array}$ & $0,80^{2}$ \\
\hline
\end{tabular}

Nota: Diferentes letras simbolizam diferenças entre as famílias ( $\mathrm{N}=$ 94); ${ }^{1}$ ANOVA: variável com distribuição normal; ${ }^{2}$ Teste $\mathrm{H}$ de Kruska/Wallis: variável com distribuição não normal.

$* p \leq 0,05$

Conforme a Tabela 2, segundo a avaliação das professoras, o tipo de família não exerceu nenhuma influência sobre as habilidades sociais das crianças e sobre a competência acadêmica infantil. Para as professoras, as crianças de famílias recasadas recentes e estáveis foram percebidas como apresentando mais problemas de comportamento externalizantes que as crianças de famílias nucleares.

\section{Discussão}

Para investigar o impacto do recasamento sobre os comportamentos e o desempenho escolar dos filhos, o presente estudo verificou, a 
partir das percepções de mães e de professoras, semelhanças e diferenças em habilidades sociais, problemas de comportamento e competência acadêmica (avaliada somente pelas professoras) de crianças de famílias nucleares e recasadas. De acordo com a Tabela 1 , nenhum indicador diferenciou as famílias pela recência do recasamento, isto é, os resultados indicaram que, segundo a percepção das mães, apenas a família nuclear se diferenciou da família recasada, independentemente do tempo de recasamento. Segundo a avaliação das mães, as crianças de famílias recasadas em transição recente e também as recasadas estáveis apresentaram menos habilidades sociais de amabilidade e autocontrole/civilidade, e mais problemas de comportamento hiperativos que as crianças de famílias nucleares. Esses resultados são diferentes dos apresentados pela literatura (Anderson \& Greene, 2013; Costa \& Dias, 2012; Sweeney, 2010) que sugere que os efeitos negativos do recasamento dos pais podem ser mais acentuados nos primeiros anos que seguem a transição familiar.

Além disso, na avaliação das mães, as crianças de famílias nucleares não apresentaram mais habilidades sociais de cooperação e autocontrole/passivo, nem menos problemas de comportamento externalizantes que as crianças cujas mães estavam recasadas há menos de três anos, mas, apenas, relativamente às crianças cujas mães estavam recasadas há mais de três anos. Tais resultados estão em desacordo com a literatura (Anderson \& Greene, 2013; Costa \& Dias, 2012; Sweeney, 2010) que afirma que o recasamento dos pais tem um efeito prejudicial imediato e temporário sobre os filhos. Contudo, esses resultados vão ao encontro do estudo de meta-análise realizado por Jeynes (2006), que identificou que a transição para o recasamento progressivamente pode se tornar uma situação crônica de estresse que afetaria negativamente o desenvolvimento social e cognitivo da criança. A criança enfrentaria várias dificuldades ao longo do tempo, tais como aceitar novo relacionamento da mãe devido a sentimentos de lealdade para com o outro progenitor (Costa \& Dias, 2012; Ramires, 2004), que ao se tornarem recorrentes no ambiente familiar fariam com que as crianças de famílias recasadas apresentassem resultados desenvolvimentais inferiores quando comparadas às crianças de famílias nucleares (Hetherington, 2003; Jensen \& Shafer, 2013, Jeynes, 2006). Uma explicação alternativa é considerar, conforme indicado por alguns estudos (Hetherington et al., 1998; King \& DeLongis, 2013; Wallersteins et al., 2013), que algumas dificuldades nos padrões relacionais não resolvidas nos primeiros anos do recasamento poderiam emergir em outras fases do ciclo vital, quando, por exemplo, os filhos passam pela transição para o primeiro ano e precisam enfrentar desafios de uma nova fase escolar. 
Porém, na avaliação das mães, crianças de famílias recasadas recentes foram mais bem avaliadas que as de famílias recasadas estáveis nas habilidades sociais infantis de cooperação e autocontrole passivo e aos problemas de comportamento externalizantes e não diferiram das crianças de famílias nucleares. Pode-se sugerir que a satisfação conjugal da mãe com o novo parceiro, verificada nos primeiros anos do recasamento pelo estudo de Hetherington (1993), poderia influenciar positivamente na sua relação com o filho, aumentando a expressão de afeto materno. É possível aludir que um efeito "lua de mel" (honeymoom), identificado na pesquisa de Hetherington (1993), esteja atenuando a percepção das mães de possíveis dificuldades no ambiente da família. Em outras palavras, a harmonia conjugal da mãe recentemente recasada com o novo parceiro a tornaria mais atenciosa e calorosa com os filhos, aumentando a chance de a mãe utilizar práticas parentais tais como expressão de afeto e estabelecimento de limites com consistência, as quais são relacionadas positivamente na literatura com as habilidades sociais dos filhos (Boas \& Bolsoni-Silva, 2010). Nesse sentido, alguns estudos encontraram que as mulheres e os homens recasados percebem a existência de padrões de relacionamento conjugal mais igualitário, identificam-se com mais paciência, flexibilidade e habilidades interpessoais para lidar com a nova e complexa dinâmica familiar (Costa \& Dias, 2012; Silva et al., 2012). Poderia haver também um esforço do parceiro da mãe em ganhar a confiança e afeto da criança, tornando-se, por exemplo, mais atencioso e expressando mais sentimentos positivos, o que poderia influenciar os comportamentos da criança, e interferir de maneira benéfica na relação do casal. Como consequência da maior disponibilidade afetiva da mãe e talvez do seu parceiro, a criança ficaria mais cooperativa e controlaria mais seus comportamentos e emoções negativas, principalmente por meio de reações encobertas, diante de discussões no ambiente da família que, nessa altura, seriam mais raras porque todos fariam esforços para se darem bem, sendo mais tolerantes uns com os outros (Costa \& Dias, 2012). Várias pesquisas vêm documentando há alguns anos que um bom relacionamento conjugal é associado positivamente a práticas parentais positivas e bem-estar psicológico dos filhos (Amato, 2005; Hetherington et al., 1998).

Outra maneira de entender as diferenças entre as famílias nucleares e recasadas estáveis é considerar que elas são configurações familiares distintas (Anderson \& Greene, 2013; Costa \& Dias, 2012; Silva et al., 2012; Sweeney, 2010). Nesse sentido, o contexto das famílias recasadas pode envolver interações idiossincráticas que são decorrentes de circunstâncias ímpares que as mesmas vivenciam após o recasamento, tais como coexistência entre parentalidade biológica e social, isto é, as relações entre a mãe biológica, o padrasto e os enteados, entre os meio-irmãos e entre os filhos de 
ambos os cônjuges que não apresentam vínculos sanguíneos (Anderson \& Greene, 2013; Silva et al., 2012).

Um fator que apoia a hipótese da especificidade do contexto do recasamento é a ausência de diferenças entre as crianças de famílias recasadas recentes e estáveis nas habilidades sociais e nos problemas de comportamento (habilidades sociais de amabilidade e autocontrole civilidade, e problemas de comportamento hiperativos) na avaliação das mães. Uma forma alternativa de se compreender a ausência de diferenças entre as crianças de famílias recasadas recentes e estáveis é considerar que as crianças que vivem em uma família recasada há mais de três anos necessitam ainda de tempo para de adaptar ao novo arranjo familiar. Há autores (Jeynes, 2006; Lima, 2008) que sinalizam que o recasamento necessitaria de mais de três anos para alcançar a estabilidade, ou seja, de três a cinco anos. Conforme Saint-Jacques et al. (2011), o cônjuge que detém a guarda dos filhos pode iniciar um novo relacionamento conjugal (recasar) antes de a família e, principalmente, os filhos, adaptaremse ao ambiente familiar pós-separação conjugal. Essa circunstância poderia demandar mais tempo, além de três anos, para os membros da família se ajustarem à nova formação familiar. Essa interpretação tem implicações para o alcance dos resultados do estudo, limitandoos. Ou seja, é possivel que as famílias recasadas há mais de três anos ainda não possam ser consideradas estáveis e não se diferenciem de forma significativa das famílias recasadas há menos de três anos.

Em relação ao contexto escolar, com exceção dos problemas de comportamento externalizantes que foram mais frequentes para as crianças de famílias recasadas recentes e estáveis, as crianças dos diferentes tipos de famílias não se diferenciaram em nenhuma das habilidades sociais investigadas nem na competência acadêmica. Resultados diferentes são sinalizados pela literatura que indica que crianças de famílias recasadas apresentaram mais dificuldades comportamentais e menos habilidades sociais do que crianças de famílias nucleares, quando avaliadas por pais (Dessen \& Szelbracikowski, 2004; Jenkins et al., 2005; Ram \& Hou, 2003; Steele, Sigle-Rushton, \& Kravdal, 2009) e professores (Jenkins et al., 2005).

Frente aos resultados, pode-se hipotetizar que os efeitos da transição familiar para o recasamento sobre os filhos sejam, para a presente amostra, mais salientes no ambiente familiar. Os ambientes da família e da escola, que juntos podem influenciar o comportamento das crianças, apresentam diferenças quanto aos estímulos, às relações e às normas de interação social. Dessa maneira, é possível que as crianças comportem-se diferentemente na família e na escola. Além disso, a ausência de diferenças nas habilidades sociais e na competência das crianças de diferentes tipos de famílias pode 
significar que as interações entre as crianças e seus colegas e suas professoras possam estar funcionando como fator de proteção nesse momento de mudança na configuração familiar, na transição para o Ensino Fundamental (Marturano, Elias, \& Leme, 2012). Assim, as crianças, em vez de estarem sobrecarregadas por essa sobreposição de transições, pelo contrário, poderiam se beneficiarn, no ambiente escolar, de oportunidades de conviver com adultos e crianças que não estariam constantemente envolvidos na resolução de problemas relacionais, conforme verificado por alguns autores (Amato, 2005, Hetherington, 2003; Jeynes, 2006).

Particularmente importante é, neste contexto, a observação da ausência de diferenças entre as crianças dos diferentes tipos de configurações familiares no que se refere à competência acadêmica, resultado que diverge da maioria dos estudos encontrados na literatura (Dessen \& Szelbracikowski, 2004; Hetherington, 2003; Steele et al., 2009). Para alguns autores (Hetherington, 2003; Jeynes, 2006; Steele et al., 2009), as mães ficariam menos disponíveis para monitorar as atividades escolares dos filhos devido aos diversos eventos estressantes que co-ocorrem junto com 0 recasamento dos pais. Contudo, alguns estudos encontraram que as crianças que frequentam os primeiros anos da escolarização são identificadas pelos professores como apresentando mais dificuldades comportamentais que acadêmicas (Lansford et al., 2006). Os resultados deste estudo parecem confirmar esta constatação. Isso pode sugerir que nos primeiros anos escolares as professoras sejam mais sensíveis às habilidades que contribuem para a adaptação das crianças às normas e às regras sociais do ambiente escolar, e mais atentas aos problemas de comportamentos externalizantes que interferem diretamente no ambiente, do que às competências acadêmicas.

De forma geral, os resultados indicaram que, segundo as avaliações das professoras, as crianças de família nuclear e recasada não apresentaram diferenças significativas em importantes habilidades sociais no contexto escolar. As habilidades sociais infantis de responsabilidade, asserção positiva, autocontrole, autodefesa e cooperação com pares avaliadas pelas professoras são importantes para o momento do desenvolvimento em que se encontravam as crianças, ou seja, a transição para o primeiro ano do ensino fundamental. Segundo Trivellato-Ferreira e Marturano (2008), a entrada no primeiro ano requer da criança novas aprendizagens de competências acadêmicas, de papéis e de habilidades de convívio social que são diferentes das demandas encontradas nos contextos da família e na pré-escola.

Todavia, os resultados indicaram maior vulnerabilidade das crianças de famílias recasadas em relação aos problemas de comportamento externalizantes, tanto no meio familiar como escolar, e às habilidades 
de cooperação, amabilidade e autocontrole e aos problemas de comportamento hiperativos, na avaliação das mães. Para Del Prette e Del Prette (2005), as habilidades sociais infantis de autocontrole e cooperação configuram-se como fator de proteção para dificuldades comportamentais e socioemocionais nos contextos da família e da escola. A habilidade social de autocontrole na infância envolve um conjunto de comportamentos que requerem da criança, por exemplo, a expressividade adequada dos seus sentimentos e capacidade de negociação em situações de conflito (Del Prette \& Del Prette, 2005). Já a habilidade social de cooperação que envolve, por exemplo, seguir regras ou instruções orais e participar de atividades em grupo, é importante para o convívio da criança na escola, em interações com pares e com professores. Assim, seria interessante que tais habilidades e dificuldades comportamentais fossem trabalhadas em programas de intervenção, seja diretamente com as crianças ou de forma indireta, com mães. Considerando que o ambiente das crianças de famílias recasadas encontra-se num momento de redefinição de papéis e funções parentais e padrões de interação, essas habilidades ajudariam as crianças a lidarem com essas mudanças, sendo mais cooperativas com pais e irmãos, expressando seus sentimentos e lidando com as situações de confronto. Concluindo, no que se refere à temporalidade dos efeitos do recasamento sobre os filhos, esta deve ser vista com cautela, especialmente em relação ao ambiente familiar que não indicou diferenças significativas nas dimensões desenvolvimentais avaliadas entre as crianças de famílias recasadas recentes e remotas. Chamam a atenção também as diferenças verificadas só entre as famílias nucleares e recasadas estáveis, com as recasadas recentes em posição intermediária, não diferindo das demais. Outros estudos poderiam investigar um número maior de famílias recasadas recentes e estáveis, fazendo variar o tempo além dos três anos. Assim, por exemplo, futuros estudos poderiam incluir um grupo de famílias recasadas há mais de cinco anos.

\section{Considerações finais}

A pesquisa possibilitou uma visão ampla e diferenciada do que seriam os efeitos dos contextos e das suas possíveis interações compensatórias, para as famílias recasadas e seus filhos que passavam pela transição para o Ensino Fundamental. Em outras palavras, se por um lado, os resultados do estudo parecem sinalizar algum impacto negativo do recasamento sobre algumas habilidades sociais e sobre os problemas de comportamento hiperativos e externalizantes de crianças de famílias recasadas em relação às crianças de famílias nucleares, especialmente no ambiente familiar, por outro, as crianças dos diferentes tipos de famílias não se 
diferenciaram, principalmente no contexto escolar, no conjunto de habilidades sociais investigadas e na competência acadêmica. Os dados indicam que as habilidades sociais, na escola, parecem não serem sensíveis às alterações no ambiente familiar. Esses resultados sugerem que os comportamentos das crianças de famílias recasadas parecem estar mais vulneráveis aos efeitos negativos do recasamento no ambiente familiar. Tais achados destacam que: (a) a compreensão do desenvolvimento infantil deve ser sempre contextualizada, levando em consideração as especificidades e idiossincrasias das relações; (b) diferentes contextos podem levar ao desenvolvimento e/ou a manifestação de diferentes comportamentos, independente da configuração familiar. Portanto, os resultados chamam a atenção para a avaliação de recursos pessoais além do ambiente da família, que podem funcionar como fatores de proteção para crianças de famílias que passam por transições e que enfrentam dificuldades em se ajustar à nova organização familiar. Além disso, o estudo se diferencia na literatura sobre recasamento, em que muitas pesquisas se baseiam num modelo de défict, isto é, investigam apenas os problemas comportamentais dos filhos. Ao tomar uma perspectiva de déficit frente ao fenômeno do recasamento, fica dificil compreender as múltiplas trajetórias que as famílias que passam por essa transição podem seguir. Nesse sentido, a presente investigação apresenta como contribuição ter investigado dimensões positivas do desenvolvimento das crianças, tais como as habilidades sociais, em dois ambientes diferentes: familiar e escolar.

O estudo apresentou algumas limitações. Primeiro, destaca-se a utilização do delineamento correlacional, que indica apenas associações entre as variáveis e não relações de precedência. O uso de metodologias de natureza longitudinal poderia ser útil para avaliar como as crianças se comportam com o passar do tempo. Segundo, a competência acadêmica das crianças foi avaliada por meio de um instrumento de medida comparativa e que se baseava nas percepções das professoras. Outros instrumentos, com avaliação objetiva de desempenho, poderiam ser utilizados. Além disso, futuros estudos poderiam avaliar as percepções dos filhos sobre as transições familiares. Finalmente, outras pesquisas poderiam investigar as representações sociais das mães, dos pais e dos professores em relação a pertencer a uma família não nuclear.

\section{Referências}

Amato, P. R. (2005). The impact of family formation change on the cognitive, social, and emotional well-Being of the next generation. The Future of Children, (15)2, 75-96. 
Anderson, E. R., \& Greene, S. M. (2013). Beyond divorce: Research on children in repartnered and remarried families. Family Court Review, 51(1), 119-130.

Associação Brasileira de Empresas de Pesquisa - ABEP. (2010). Critério de classificação econômica Brasil, 2010. Dados com base no levantamento socioeconômico, IBOPE, 2000. Recuperado em 17 de junho, 2010, de http://www.abep.org.br. Acesso em: 17 de jun. de 2010.

Bandeira, M., Del Prette, Z. A. P., Del Prette, A., \& Magalhães, T. (2009). Validação das escalas de habilidades sociais, comportamentos problemáticos e competência acadêmica (SSRS-BR) para o ensino fundamental. Psicologia: Teoria e Pesquisa, 25(2), 271-282.

Boas, A. C. V. V., \& Bolsoni-Silva, A. T. (2010). Habilidades sociais educativas de mães separadas e sua relação com comportamento de pré-escolares. Psico-USF, 15(3), 301-310.

Booth, A., \& Amato, P. R. (2001). Parental predivorce relations and offspring postdivorce well-being. Journal of Marriage and the Family, 63(1), 197-212.

Caballo, V. E. (2010). Manual de avaliação e treinamento das habilidades sociais (S. M. Dolinsky, Trad.). São Paulo: Santos.

Cadima, J., McWilliam, R. A., \& Leal, T. (2010). Environmental risk factors and children's literacy skills during the transition to elementary school. International Journal of Behavioral Developmental, 34(1), 24-33

Cano, D. S., Gabarra, L. M., Moré, C. O., \& Crepaldi, M. A. (2009). As transições familiares do divórcio ao recasamento no contexto brasileiro. Psicologia: Reflexão e Crítica, 22(2), 214-222.

Costa, J. M., \& Dias, C. M. de S. B. (2012). Famílias recasadas: mudanças, desafios e potencialidades. Psicologia: Teoria e Pesquisa, 14(3), 72-87.

Del Prette, Z. A. P., \& Del Prette, A. (2005). Psicologia das habilidades sociais na infância: teoria e prática. Petrópolis: Vozes.

Dessen, M. A., \& Szelbracikowski, A. C. (2004). Crianças com problemas de comportamento exteriorizado e dinâmica familiar. Interação em Psicologia, 8(2), 171-180.

Hetherington, E. M. (1993). An overview of the Virginia longitudinal study of divorce and remarriage with a focus on early adolescence. J ournal of Family Psychology, 2(1), 39-56.

Hetherington, E. M. (2003). Intimate pathways: Changing patterns in close personal relationships across time. Family Relations, 52(4), 318-331.

Hetherington, E. M., Bridges, M., \& Insabella, G. M. (1998). What matters? What does not? Five perspectives on the association 
between marital transitions and children's adjustment. American Psychologist, 53(2), 167-184.

Hoglund, W. L., \& Leadbeater, B. J. (2004). The effects of family, school, and classroom ecologies on changes in children's social competence and emotional and behavioral problems in first grade. Developmental Psychology, (40)4, 533-544.

Instituto Brasileiro de Geografia e Estatística - IBGE (2010). Estatística do Registro Civil 2010. Recuperado em 13 de maio, 2011, de http://www.ibge.gov.br/home/estatistica/populacao/registrocivi 1/2010/default.shtm

Jeynes, W. H. (2006). The impact of parental remarriage on children: a Meta-analysis. Marriage and Family Review, 40(4), 75-102.

Jenkins, J., Simpson, A., Dunn, J., Rasbash, J., \& O'Connor, T. G. (2005). Mutual influence of marital conflict and children's behavior problems: Shared and nonshared family Risks. Child Development, 76(1), 24-39.

Jensen, T. M., \& Shafer, K. (2013). Stepfamily functioning and closeness: Children's view on second marriages and stepfather relationships. Social Work, 58(2), 127-136.

King, D. B., \& DeLongis, A. (2013). Dyadic coping with stepfamily conflict: Demand and withdraw responses between husbands and wives. Journal of Social and Personal Relationships, 30(2), 198-206.

Lansford, J. E., Malone, P. S., Castellino, D. R., Dodge, K. A., Pettit, G. S., \& Bates, J. E. (2006). Trajectories of internalizing, externalizing, and grades for children who have and have not experienced their parents' divorce or separation. Journal of Family Psychology, 20(2), 292-301.

Lima, I. B. (2008). Família reconstituída: A nova construção da conjugalidade. Revista de Enfermagem, 2(3), 269-278.

Marôco, J. (2011). Análise estatística com a utilização do SPSS. 5a Ed. Lisboa: ReportNumber.

Marturano, E. M. (2008). Tensões cotidianas na transição da primeira série: Um enfoque de desenvolvimento. Psicologia em Estudo, 13(1), 77-85.

Marturano, E. M., Elias, L. C. Dos S., \& Leme, V. B. R. (2012). A família e o desenvolvimento do escolar. In: L. E. Melchiori, O. M. P. R. Rodrigues, \& A. C. B. Maia (Orgs.), Família e crianças: reflexões teórico-práticas sobre os cuidados com as crianças (pp. 137-153). Curitiba: Juruá.

McGoldrick, M., \& Carter, B. (2008). Constituindo uma família recasada. Em B. Carter \& McGoldrick, M. (Orgs.), As Mudanças no Ciclo de Vida Familiar: Uma estrutura para a terapia familiar (pp. 291-321). 2a ed. Tradução Maria Adriana Veríssimo 
Veronese. Porto Alegre: Artmed. (Trabalho original publicado em 1989).

Oliveira, D. de, Siqueira, A. C., Dell'Aglio, D. D., \& Lopes, R. de C. S. (2008). Impacto das configurações familiares no desenvolvimento de crianças e adolescentes: uma revisão da produção científica. Interação em Psicologia, 12(1), 87-98.

Ramires, V. R. R. (2004). As transições familiares: A perspectiva de crianças e pré-adolescentes. Psicologia em Estudo, 9(2), 183193.

Ram, B., \& Hou, F. (2003). Changes in family structure and child outcomes: Roles of economic and familial resources. The Policy Studies J ournal, 31(3), 309-330.

Saint-Jacques, M-C., Robitaille, C., Godbout, E., Parent, C., Drapeau, S., \& Gagne, M.H. (2011). The processes distinguishing stable from unstable stepfamily couples: A qualitative analysis. Family Relations, 60(5), 545-561.

Silva, P. De O. M., Trindade, Z. A., \& Junior, A. Da. S. (2012). As representações sociais de conjugalidade entre casais recasados. Estudos de Psicologia, 17(3), 435-443.

Stasiak, G. R., \& Weber, L. N. D. (2013). Percepção do estresse pelas crianças do primeiro ano do Ensino Fundamental. Impulso, 23(56), 35-45.

Steele, F., Sigle-Rushton, W., \& Kravdal, O. (2009). Consequences of family disruption on children's educational outcomes in Norway. Demography, 46(3), 553-574.

Sweeney, M. M. (2010). Remarriage and stepfamilies: Strategic sites for family scholarship in the $21^{\text {st }}$ century. Journal of Marriage and Family, 72(3), 667-684.

Trivellato-Ferreira, M. de. C. \& Maturano, E. M. (2008). Recursos da criança, da família e da escola predizem competência na transição da $1^{a}$ série. Revista Interamericana de Psicología, 42(2), 549-558.

Wallersteins, J., Lewis, J., \& Rosenthal, S. P. (2013). Mothers and their children after divorce: Report from a 25-year longitudinal study. Psychoanalytic Psychology, 30(2), 167-187.

\section{Endereço para correspondência \\ Vanessa Barbosa Romera Leme}

Rua Marechal Deodoro, 263, Centro, CEP 24030-060, Niterói - RJ, Brasil

Endereço eletrônico: vanessaromera@gmail.com

\section{Edna Maria Marturano}

Av. Bandeirantes, 3900, Jardim Recreio, CEP 14049-900, Ribeirão Preto - SP, Brasil Endereço eletrônico: emmartur@fmrp.usp.br

\section{Anne Marie Germaine Victorine Fontaine}

Rua Dr. Alfredo Allen, 4200-135 - Porto, Portugal

Endereço eletrônico: fontaine@fpce.up.pt 
Recebido em: 02/06/2014

Reformulado em: 01/09/2014

Aceito para publicação em: 02/09/2014

\section{Notas}

* Professora do Programa de Pós-Graduação em Psicologia da Universidade Salgado de Oliveira - Universo, Niterói - RJ

** Professora Titular da Faculdade de Medicina da Universidade de São Paulo FMRP, USP, Ribeirão Preto - SP

*** Professora Catedrática da Faculdade de Psicologia e Ciências da Educação da Universidade do Porto - FPCEUP, Porto, Portugal

${ }^{1}$ Pesquisa financiada pela Fundação de Amparo à Pesquisa do Estado de São Paulo (FAPESP - Processo n. 52132-08) 